\title{
Proposal of an educational pattern house to be powered using renewable energies
}

Rico-Secades, Manuel (http://orcid.org/000-0002-5372-0330); Calleja-Rodríguez, Antonio Javier (http://orcid.org/0000$\underline{0002-4271-8227})$.

Abstract.- This work has educational purpose and aims at defining and standardizing a pattern home for engineering students can incorporate renewable energy to meet the energy needs of the house and propose new concepts and services linked to power electronics, renewable energies and energy efficiency.

It has been chosen a real location in the university Campus of Gijon for the purpose of standardizing weather conditions (to define needs for heating and DHW) and also simplify the definition of renewable energy resources available (both solar and wind energies).

Engineering students on the issues of Power Electronics and Renewable Energy will have a well-defined framework on which to apply different technologies: DHW solar, thermal foil for heating, lighting based on LEDs for outdoor and indoor situations, energy storage, energy self-consumption, net-metering, charging stations for electric vehicles (EV), smart furniture, strategies smart grid, DC distribution, energy saving strategies and many others that are open to the imagination and initiative of the students.

Index Terms.- Renewable energy, solar energy, wind energy, Heating, domestic hot water (DHW), energy efficiency, power electronics, smart grid, Smart cities, led lighting, engineering education.

Affiliation: All authors are from EPI Gijón. Electrical Engineering Department. Campus de Viesques- Building 3 - ES33204 - GIJON - ASTURIAS - SPAIN.

E-mail of authors: Rico-Secades M. is the corresponding author (mrico@uniovi.es), 


\section{INTRODUCCIÓN}

Se propone una vivienda hipotética con un propósito puramente educacional (Educational Pattern House$\boldsymbol{E P H}$ ), la cual dispone de unas condiciones geométricas y funcionales previamente prefijadas, unas posibilidades concretas y está situada en una ubicación geográfica real. De esta forma las necesidades de calefacción, de agua caliente sanitaria (ACS) (en inglés: DHW-Domestic Hot Water), el recurso solar y eólico disponible y otras muchas características están previamente definidas y estandarizadas, de una forma simplificada y con un fin puramente didáctico.

Los estudiantes pueden así usar este elemento predefinido para aplicar sobre el diferentes técnicas y conocimientos en un contexto de aprendizaje basado en proyectos (PBL - Project Based Learning $)$ aplicado a los campos de la Electrónica de Potencia, la Eficiencia Energética y las Energías Renovables. Se trata de una iniciativa del Taller de Energías Renovables (WRE-Workroom on Renewable Energy) que se ha puesto en marcha en la Escuela Politécnica de Ingeniería de Gijón (EPI-Gijón). Un ejemplo de estos proyectos realizados por estudiantes puede encontrarse en la referencia [4], en este caso, realizado sobre una vivienda real propuesta por el propio estudiante.

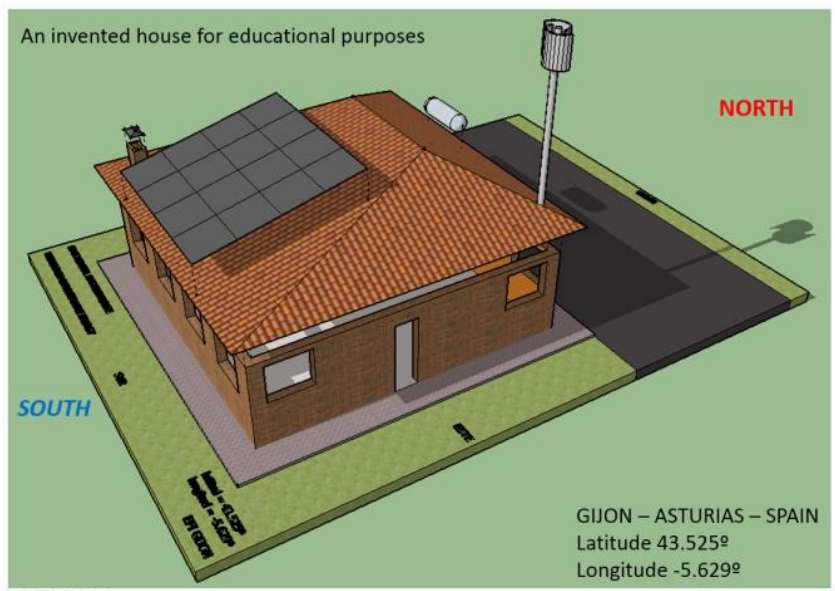

Fig. 1. Una vista general de la vivienda patrón educacional propuesta "Educational Pattern House (EPH)".

Este artículo se describe la vivienda, su ubicación y dimensiones geométricas, estandariza los coeficientes de transmisión de calor de todos los elementos de forma que las necesidades energéticas de la vivienda patrón queden pre-definidas para todos los estudiantes de forma idéntica. La figura 1 muestra una vista general de la vivienda patrón educacional (EPH) propuesta con todos sus elementos. 


\section{DESCRIPCION DE LA VIVIENDA PATRÓN EDUCAIONAL Y TODOS SUS ELEMENTOS}

En primer lugar se establece una ubicación real para la vivienda patrón educational (EPH), así se ha seleccionado el Campus Universitario de Gijón como ubicación. La figura 2 recoge los detalles y coordenadas geográficas de la ubicación seleccionada.

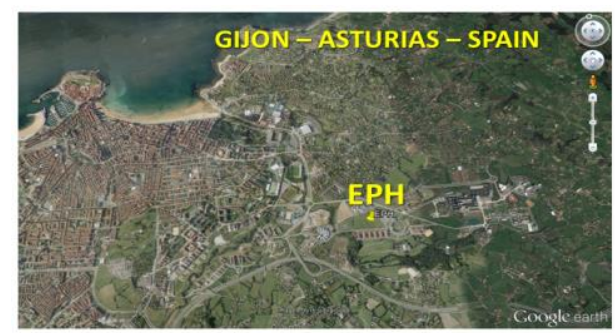

GIJON - ASTURIAS - SPAIN

Latitude 43.525 은

Longitude -5.629 은

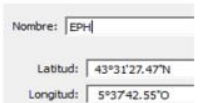

Longlud: $\longdiv { 5 0 3 7 4 2 . 5 5 ^ { \circ } \mathrm { O } }$

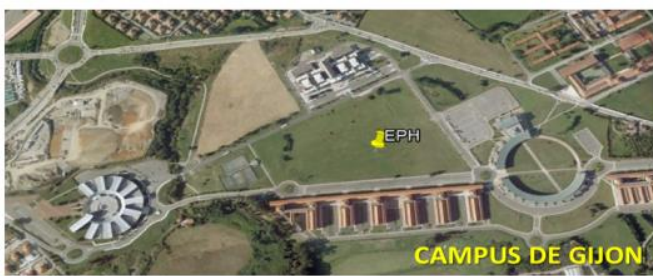

Fig. 2. Ubicación de la vivienda patrón educacional (EPH)

La vivienda patrón educacional $(\mathrm{EPH})$ cuenta con una planta de $10 \mathrm{~m}$ x $10 \mathrm{~m}\left(100 \mathrm{~m}^{2}\right)$ y una altura de paredes de $3 \mathrm{~m}$. Cuenta con 12 ventanas y dos puertas tal y como se recoge en detalle en las figuras 3, 4, 5, 6 y 7. En estas figuras se muestran los detalles de cada una de las fachadas de la vivienda patrón educacional (EPH) indicando también los coeficientes de transmisión térmica $(\mathrm{K})$ de cada uno de estos elementos en vista al dimensionado posterior de la calefacción de la vivienda.

Notar que para las unidades del coeficiente transmisión térmica de todos los elementos hemos tomado deliberadamente las unidades $\mathrm{Wh} / \mathrm{h} \mathrm{m}^{2}{ }^{\circ} \mathrm{C}$, queriendo dejar claro el sentido físico de la pérdida de energía en Wh cada hora. (En las bibliografía y referencias técnicas se emplea habitualmente $\mathrm{W} / \mathrm{m}^{2}{ }^{\circ} \mathrm{C}$ ). 

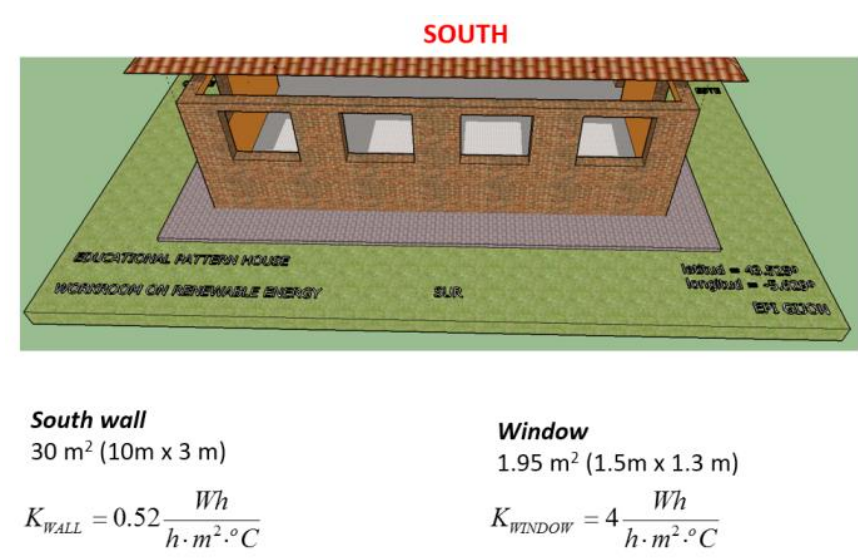

Fig. 3. Fachada Sur de la vivienda patrón educacional (EPH)

Las ventanas son todas idénticas de $1.5 \mathrm{~m}$ x $1.3 \mathrm{~m}$ con un coeficiente de transmisión térmica de $4 \mathrm{Wh} / \mathrm{h} \mathrm{m}^{2}{ }^{\circ} \mathrm{C}$ que equivaldría aproximadamente a unas ventanas con doble cristal. Para el muro exterior hemos considerado un coeficiente de pérdidas de $0.52 \mathrm{Wh} / \mathrm{h} \mathrm{m}^{2}{ }^{\circ} \mathrm{C}$, que se corresponde con un muro con unos $6 \mathrm{~cm}$ de aislamiento térmico.

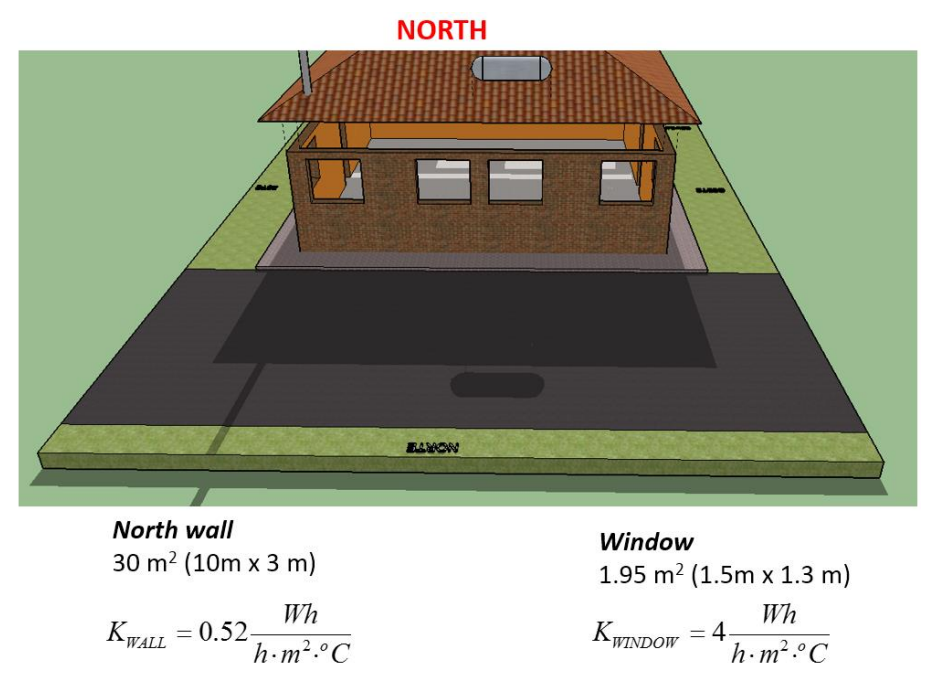

Fig. 4. Fachada Norte de la vivienda patrón educacional (EPH)

En las fachadas Este y Oeste hemos previsto dos puertas de $2.5 \mathrm{~m} \mathrm{x} 0.8 \mathrm{~m}$ con un coeficiente de transmisión térmica de $5 \mathrm{Wh} / \mathrm{h} \mathrm{m}^{2}{ }^{\circ} \mathrm{C}$ (un peor aislamiento térmico que para las ventanas). 
EAST

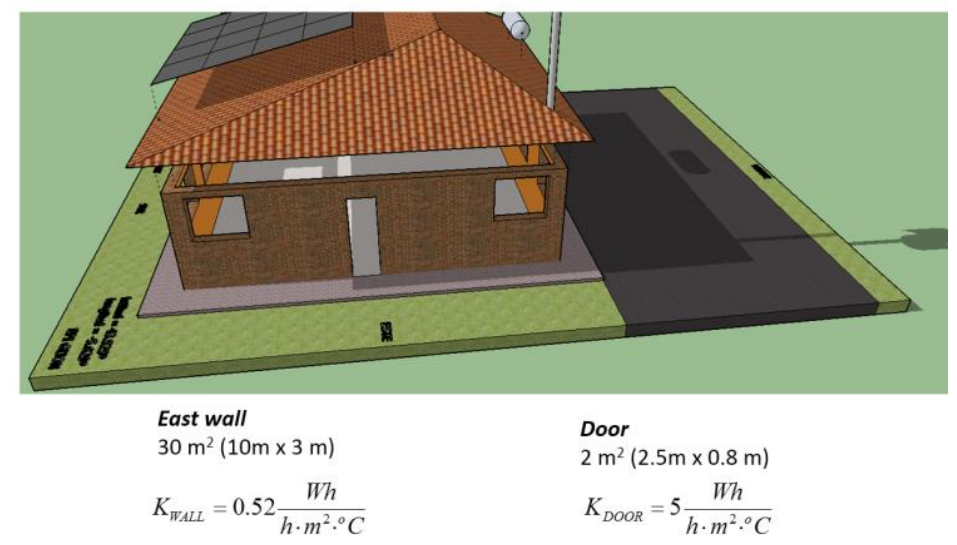

Fig. 5. Fachada Este de la vivienda patrón educacional (EPH)

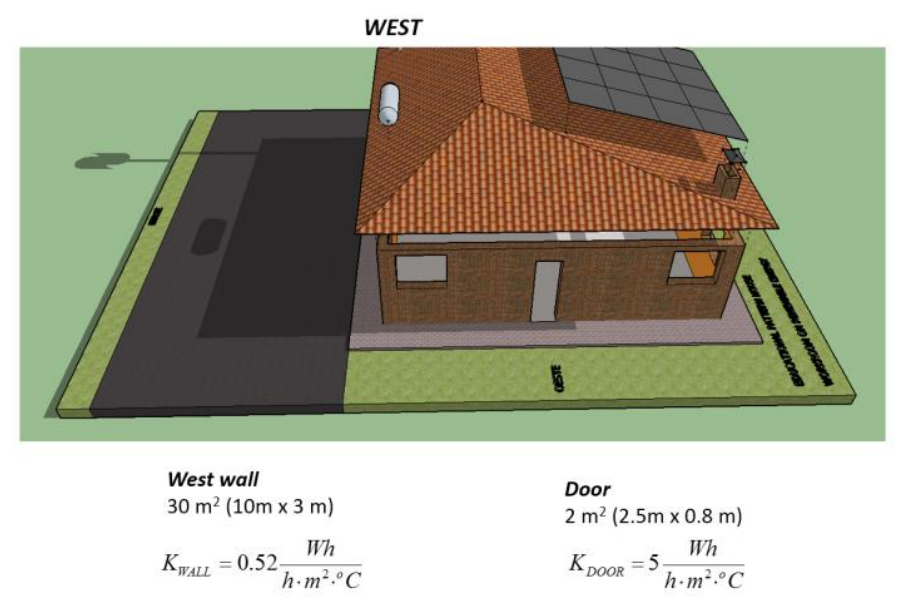

Fig. 6. Fachada Oeste de la vivienda patrón educacional $(E P H)$

El suelo de la vivienda patrón educacional (EPH) se ha elevado $0.5 \mathrm{~m}$ sobre el suelo para disponer de una cámara donde instalar calefacción eléctrica mediante lámina radiante, una de las propuestas que se presenta a los estudiantes de ingeniería. En este sentido, el aislamiento térmico del suelo se ha considerado bastante bueno $\left(0.5 \mathrm{Wh} / \mathrm{h} \mathrm{m}^{2}{ }^{\circ} \mathrm{C}\right)$. El tejado se ha preparado con una amplia pendiente orientada al sur, para colocar paneles solares y con un mástil metálico preparado para la incorporación de un pequeño aerogenerador eólico. En ambos casos, propuestas que quedan abiertas al diseño e iniciativa de los estudiantes. El aislamiento térmico del techo se ha considerado pues bastante reducido $\left(0.5 \mathrm{Wh} / \mathrm{h} \mathrm{m}^{2}{ }^{\circ} \mathrm{C}\right)$. 


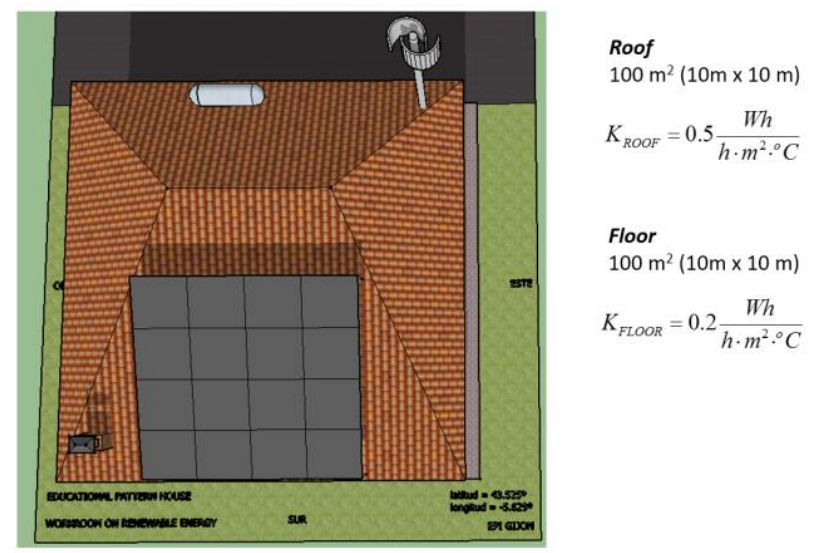

Fig. 7. Techo y suelo de la vivienda patrón educacional (EPH)

La superficie prevista para paneles solares es de $5.98 \mathrm{~m}$ x $5.58 \mathrm{~m}$, está orientada al sur (Azimuth $=0^{\circ}$ ) y con la inclinación (Slope) regulable. Los estudiantes pueden optar por utilizar esta superficie para colocar el número de paneles que estimen oportuno, con la inclinación deseada y configuración que decidan. Los detalles geométricos de la zona dispuesta para paneles solares se muestran en la figura 8 .

Lo mismo sucede con el mástil previsto para la colocación de un pequeño aerogenerador a una altura de $10 \mathrm{~m}$. Los detalles de este mástil están recogidos en la figura 9. De igual manera, los estudiantes pueden realizar propuestas de diseño de eje vertical (VAWT) o de eje horizontal (HAWT), con dimensiones acordes a las dimensiones disponibles, con el diseño y estética que el propio estudiante estime oportuno.

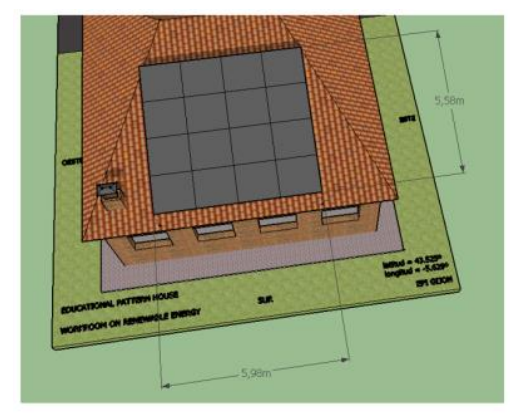

PV Solar surface

$33.368 \mathrm{~m}^{2}(5.98 \mathrm{~m} \times 5.58 \mathrm{~m})$

Azimuth 0 (South)

Adjustable Slope

Fig. 8. Superficie prevista para paneles solares en la vivienda patrón educacional (EPH)

Finalmente, en la vivienda patrón educacional (EPH) se ha previsto un depósito para agua caliente sanitaria (ACS) (Domestic Hot Water - DHW en inglés) para satisfacer las necesidades de la vivienda. Se ha considerado para el mismo un radio de $0.3 \mathrm{~m}$ y una altura de $1.5 \mathrm{~m}$ con un aislamiento térmico fijado en $0.5 \mathrm{Wh} / \mathrm{h} \mathrm{m}^{2}{ }^{\circ} \mathrm{C}$. 


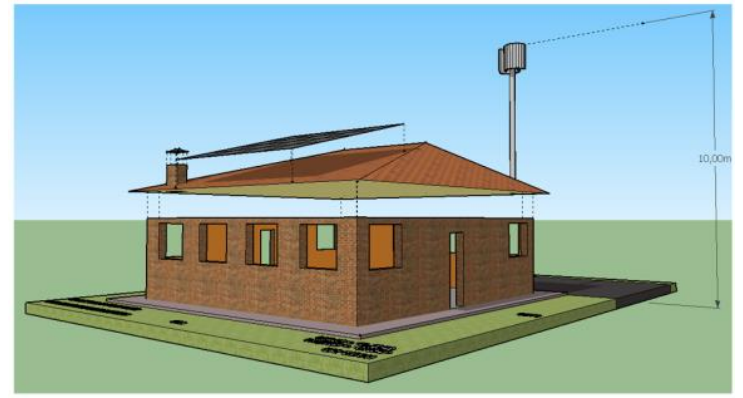

Fig. 9. Mástil para pequeño generador eólico en la vivienda piloto (EPH)

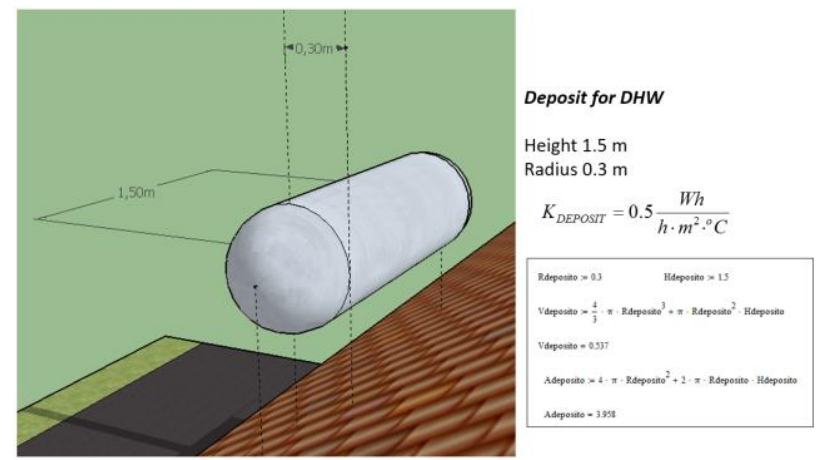

Fig. 10. Depósito para ACS en la vivienda patrón educacional (EPH)

\section{NECESIDADES DE CALEFACCIÓN Y PROPUESTAS DE TRABAJO RELACIONADAS}

Definidos los coeficientes de transmisión térmica de la vivienda patrón educacional (EPH) las pérdidas de calor en la vivienda pueden ser fácilmente calculadas y dependientes de la temperatura exterior en cada momento. La figura 11 muestra así una gráfica con las pérdidas energéticas horarias en función la temperatura externa y asumiendo una temperatura interna objetivo de $20^{\circ} \mathrm{C}$.

Puede obtenerse fácilmente que para una temperatura exterior extrema fijada en $-5^{\circ} \mathrm{C}$, las pérdidas de calor son del orden de $5 \mathrm{kWh}$, es decir, que un aporte de potencia térmica de $5 \mathrm{~kW}$ constante durante esta hora permitiría compensar está perdida energética y, por ende, esto permite dimensionar la potencia máxima necesaria para la calefacción en condiciones extremas. 


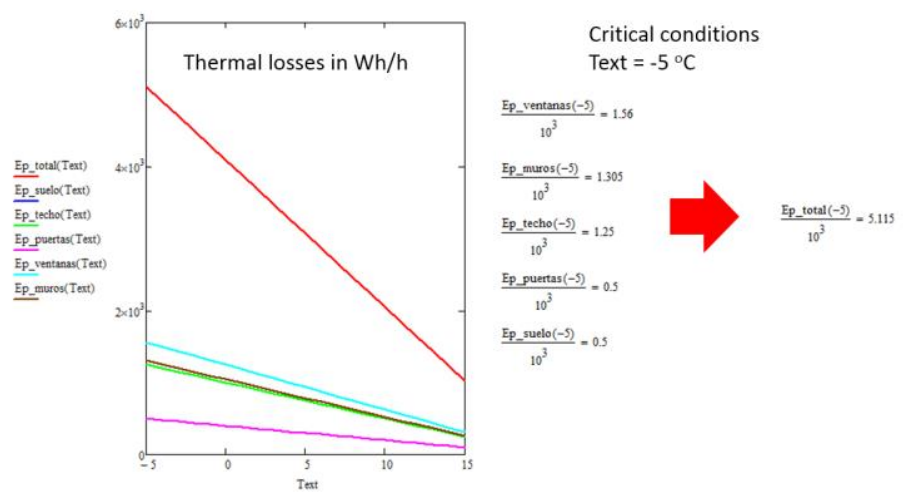

Fig. 11. Pérdidas de energía en la vivienda en función de la temperatura exterior.

Como curiosidad se ha calculado el porcentaje de pérdidas de calor en la vivienda, pudiendo observarse que ventanas y puertas son elementos críticos que juntos representan más del 50\% de las mismas, dato que coincide con la situación de viviendas reales, siendo este uno de los elementos clave en las estrategias de ahorro de energía en las viviendas.

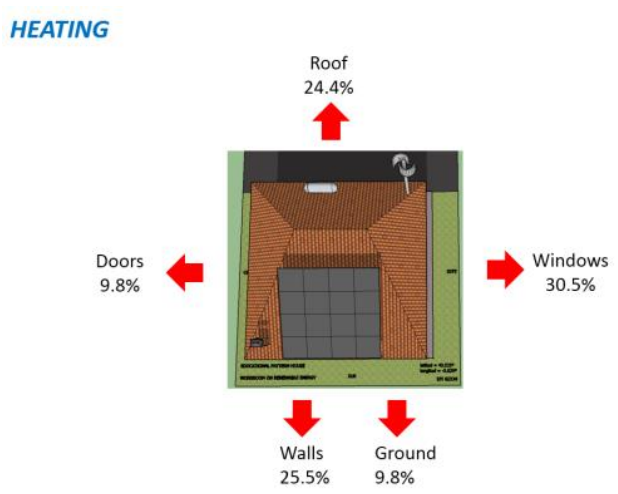

Fig. 12. Porcentajes de pérdidas en los distintos elementos de la vivienda EPH.

La estimación de las necesidades de calefacción mensuales y anuales, es complicada y depende de las condiciones climáticas de la zona donde esté instalada la vivienda. Para facilitar y estandarizar el trabajo de los estudiantes se ha considerado una variación sinusoidal de la temperatura diaria, considerando un día promedio de cada mes del año a partir de los datos de las temperaturas medias mínimas y máximas de la ciudad de Gijón.

Esto nos permite determinar de una forma aproximada las necesidades de calefacción de cada mes del año. La 
figura 13, recoge los datos utilizados del modelo para cada mes y una representación gráfica para el mes de enero (uno de los meses más fríos del año).

HEATING

Model used for daily outdoor temperature fluctuation

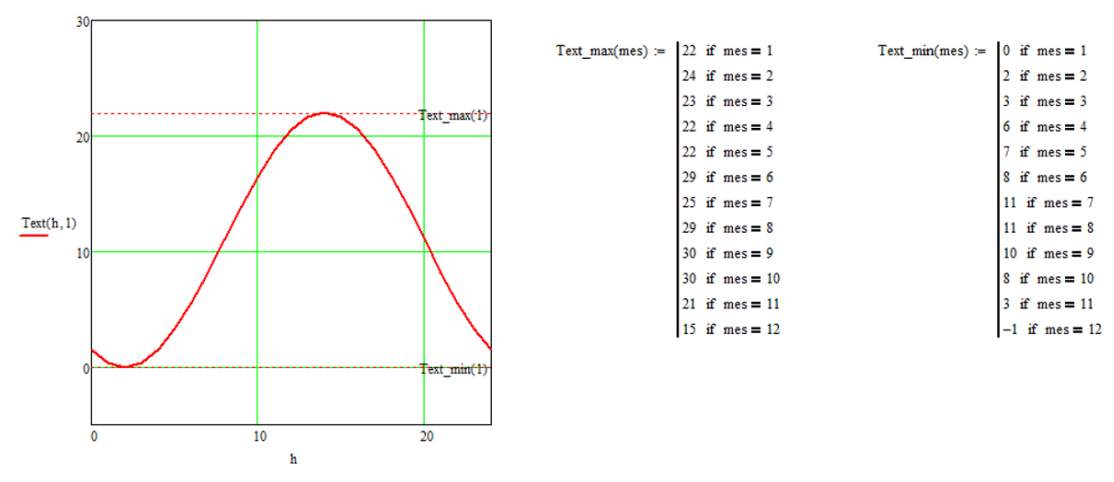

Fig. 13. Modelo utilizado para la temperatura exterior diaria de un día promedio de cada mes del año.

Utilizando estos datos se puede establecer un criterio para decidir qué meses del año se debe de encender la calefacción y cuáles no, a partir de los datos de la temperatura promedio del día tipo de cada mes.

La figura 14 muestra los datos obtenidos y la decisión de no considerar la calefacción encendida (a efectos de cálculos energéticos) los meses comprendidos entre el mes 6 (junio) y el mes 10 (octubre) ambos incluidos.

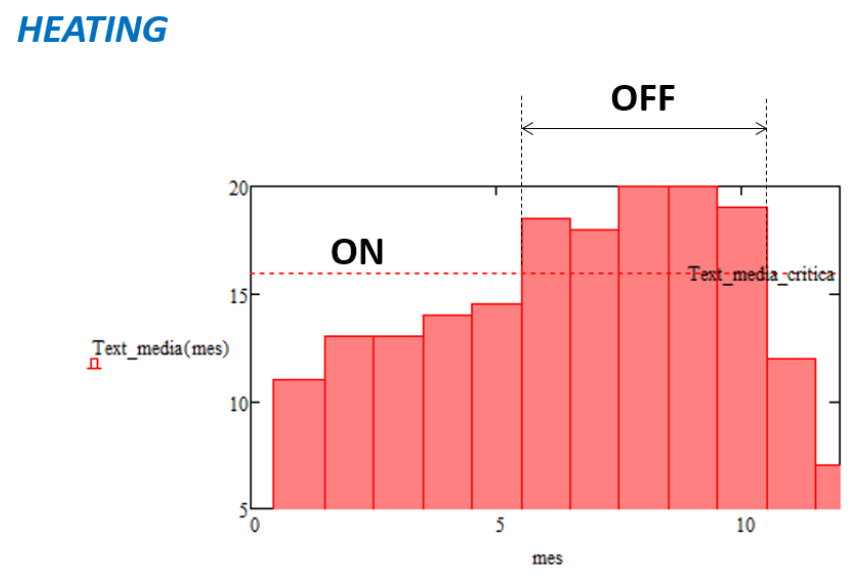

Fig. 14. Meses con calefacción y meses sin ella.

Con estos datos ya podemos fácilmente calcular las necesidades energéticas mensuales y anuales de calefacción. 
Así la figura 15, muestra el consumo de un día tipo de cada mes, el total mensual y el total anual (8.3 MWh/año).
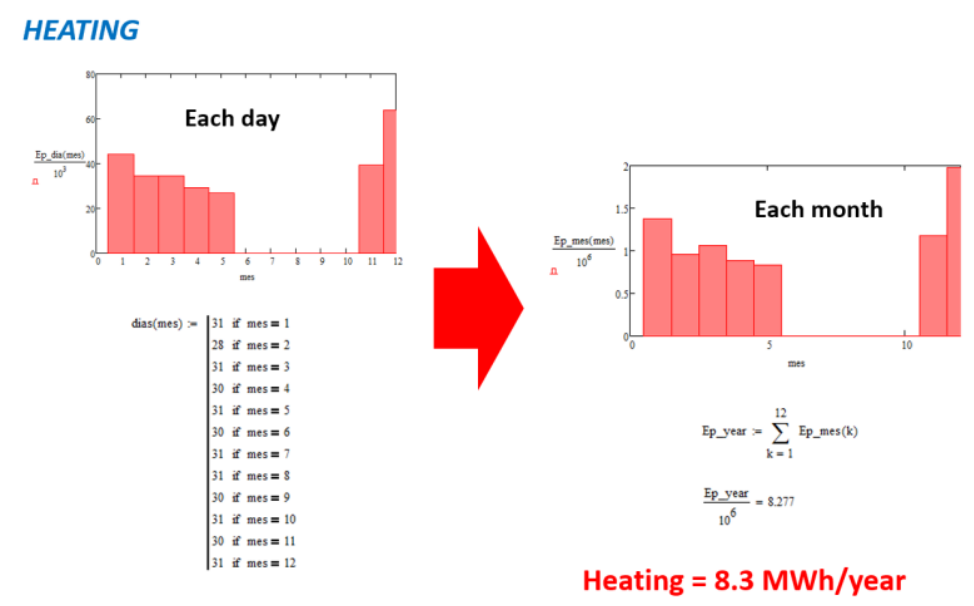

Fig. 15. Necesidades energéticas mensuales y anuales de calefacción.

Todo esto, deja abierta la puerta a las propuestas de los estudiantes para satisfacer esta demanda energética utilizando energías renovables (bien sea solar o eólica). A modo de ejemplo la figura 16 recoge una estimación del coste anual de esta energía suponiendo consumo eléctrico, la cantidad equivalente de Gas Natural (GN) y los $\mathrm{Kg}$ de $\mathrm{CO}_{2}$ emitidos a la atmosfera en caso de utilizar este tipo de recurso energético. Quedan para los estudiantes otros cálculos y propuestas que se estimen oportunas.

\section{HEATING}
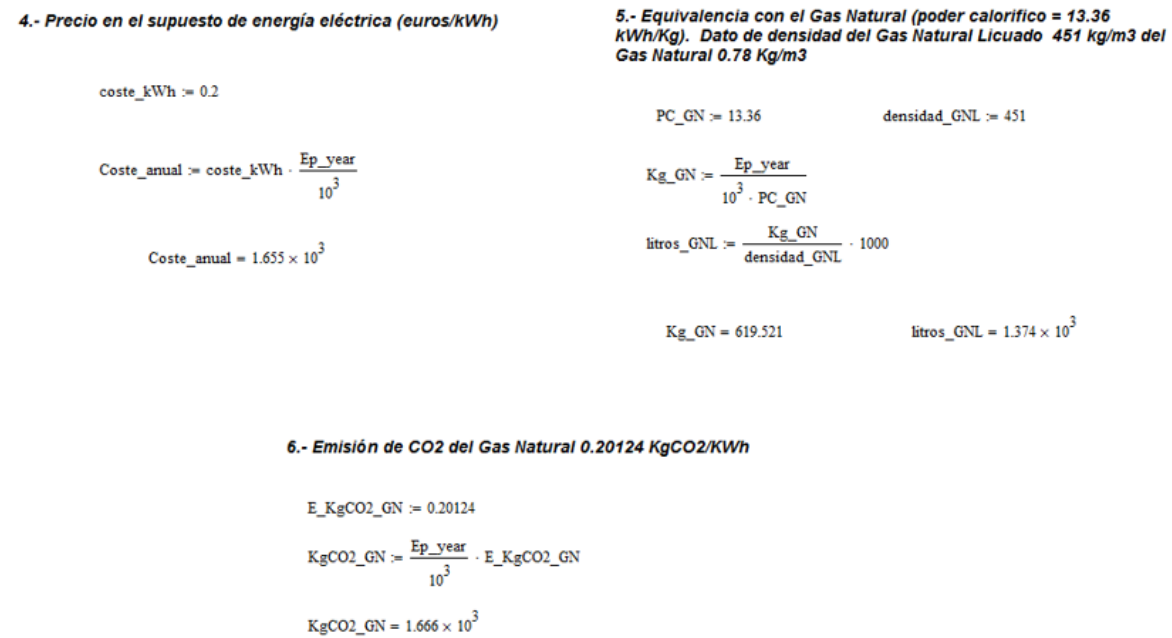

Fig. 16. Equivalente económico para el caso de energía eléctrica y necesidades de gas natural (GN) y emisiones de CO2 para este caso.

Entre las propuestas que se sugieren a los estudiantes, está el uso de lámina térmica radiante (Heating Film) de 
última generación (como alternativa al hilo radiante) como elemento calefactor principal. Utilizando este elemento se sugieren bien estrategias desde red (alimentación en alterna con control de potencia, autoconsumo solar y/o eólico y técnicas de balance neto, "net-metering" en inglés) o estrategias desde bus de continua con incorporación de energías renovables y almacenamiento de energía (vivienda aislada).

Estrategias tipo red inteligente en continua (DC Smart Grid), técnicas de eficiencia energética, reducción de pico de potencia, distribución y ecualización de la potencia, calefacción selectiva por zonas, etc quedan a la iniciativa del estudiante. La figura 17 recoge una pequeña panorámica de estas opciones expuestas.

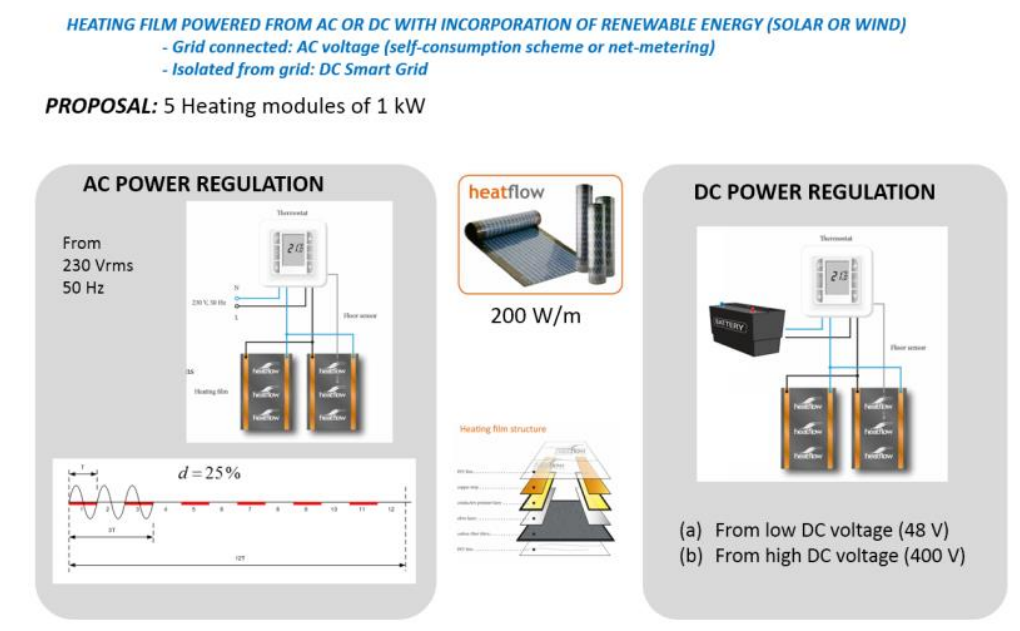

Fig. 17. Propuestas de calefacción a partir de lámina térmica radiante (Heating Film).

Este tipo de lámina térmica radiante se considera también interesante para que el estudiante pueda incorporar propuestas de elementos de mobiliario urbano que incorporen capacidad calefactora, de corta duración y potencia limitada. Se deja la propuesta lanzada y abierta a la creatividad de estudiante en este aspecto.

\section{AGUA CALIENTE SANITARIA Y PROPUESTAS DE TRABAJO RELACIONADAS}

Para el caso del agua caliente sanitaria (por sus siglas en español ACS o en Inglés: Domestic Hot Water DHW) se han estimado las necesidades de la vivienda EPH. Se ha considerado la temperatura promedio del agua fría de cada mes y un consumo mensual de agua caliente de 200 litros/día a una temperatura de $60^{\circ} \mathrm{C}$.

La figura 18 recoge los datos de energía necesaria para las necesidades de ACS en un día tipo de cada mes y el total mensual y anual necesario. Siguiendo una analogía con los datos de la calefacción se ha obtenido el coste 
económico en el caso de utilizar electricidad y la equivalencia en Gas Natural (GN) y sus correspondientes emisiones de $\mathrm{CO}_{2}$ a la atmosfera (figura 19).

DOMESTIC HOT WATER (DHW)

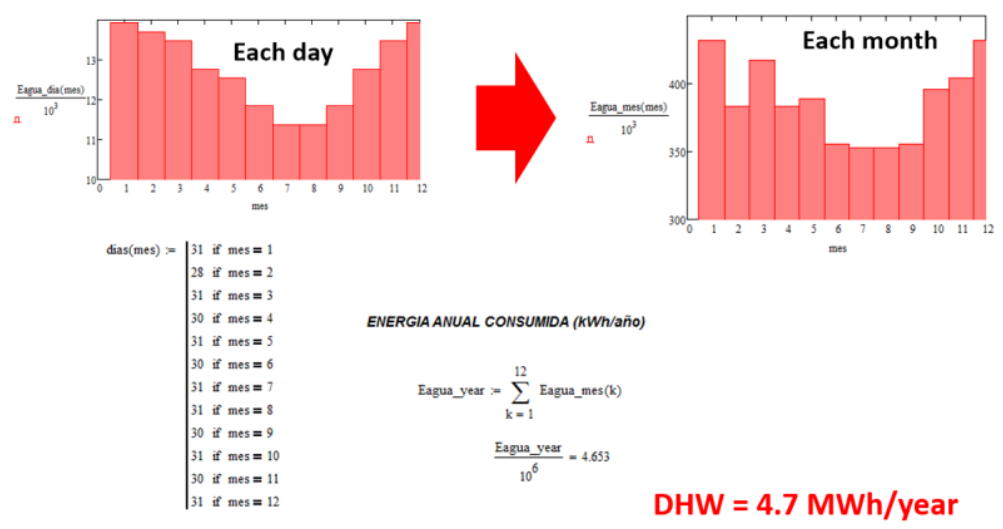

Fig. 18. Necesidades energéticas para agua caliente sanitaria (ACS).

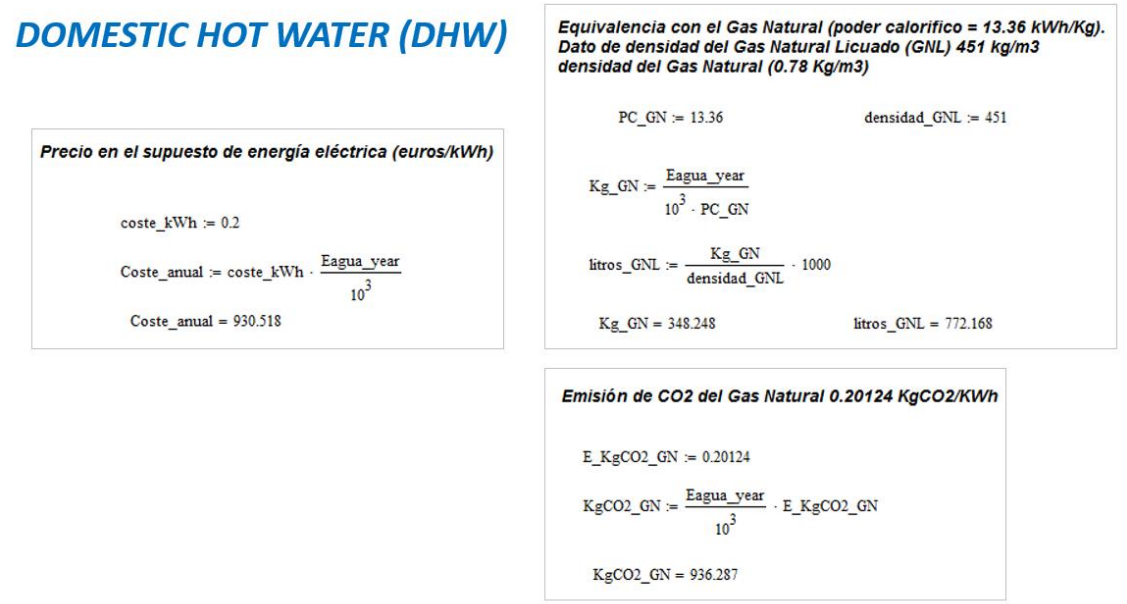

Fig. 19. Coste energía eléctrica, equivalencia con GN y emisiones de CO2 para ACS.

Pasa satisfacer esta demanda de ACS, la vivienda piloto educacional (EPH) se ha provisto de un depósito de una capacidad de unos 500 litros de agua cuyas dimensiones y datos ha han sido reflejados en la figura 10. Por dar un dato de referencia al estudiante para que haga sus propios cálculos, la energía necesaria para un día típico del mes de enero (unos $14 \mathrm{kWh}$ ) puede aportarse con una resistencia eléctrica de $2.3 \mathrm{~kW}$ a plena potencia durante 6 horas.

Considerando los mismos datos ambientales utilizados para el cálculo de la calefacción y lo datos del coeficiente 
de pérdidas del depósito pueden estimarse las pérdidas de energía del mismo, suponiendo que logramos mantenerlo lleno de agua a $60^{\circ} \mathrm{C}$ durante todo el año.

La figura 20 recoge los resultados obtenidos para las pérdidas caloríficas en el depósito para un día tipo de cada mes y también los datos mensuales y anuales respectivamente. Los cálculos económicos que representan estas pérdidas de calor en el caso de utilizar energía eléctrica, las equivalencias con Gas Natural (GN) y las emisiones de $\mathrm{CO}_{2}$ a la atmosfera, se dejan en este caso para el trabajo de los estudiantes.

Como dato de referencia puede estimarse que para una temperatura extrema exterior de $-5^{\circ} \mathrm{C}$ las perdidas horarias de calor en el depósito son de unos $124 \mathrm{Wh}$, que permiten hacerse una idea de la potencia necesaria para compensar esta pérdida energética en cada momento.

DEPOSIT FOR DOMESTIC HOT WATER (DHW)

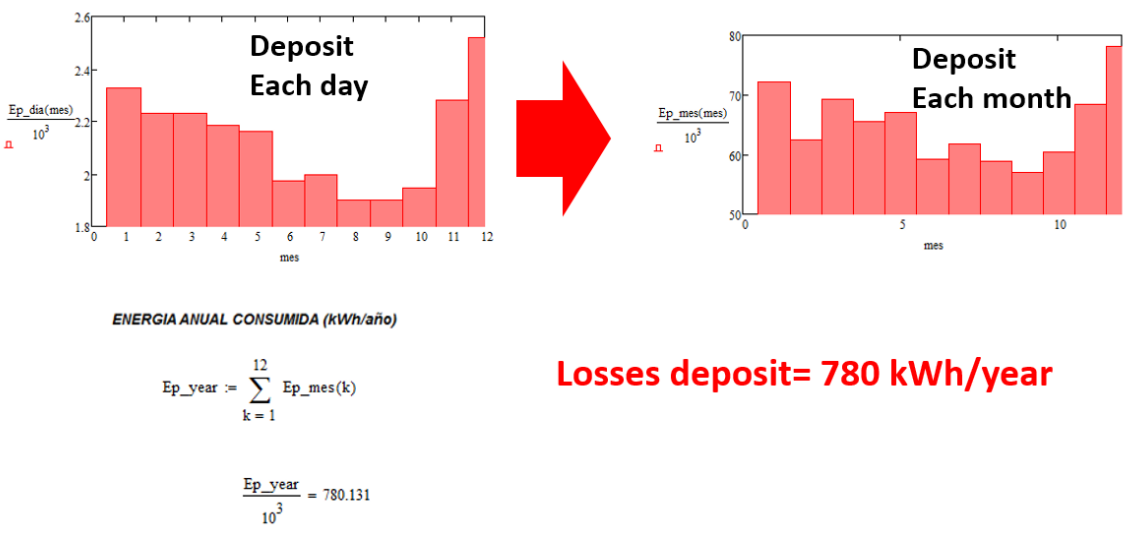

Fig. 20. Energy losses in deposit for DHW.

Así las propuestas de trabajo relacionadas con este punto están ligadas a los temas de respaldo solar o eólico a la energía eléctrica necesaria para satisfacer la demanda de ACS de la vivienda EPH (sistemas conectados a red), también puede plantarse el respaldo a sistemas de Gas Natural o equivalentes (Gasoil, Calderas de Biomasa, etc).

En otra línea de trabajo estarían los sistemas aislados con almacenamiento de energía en baterías, para satisfacer de forma autosuficiente las necesidades energéticas para ACS utilizando únicamente energía eléctrica de fuente renovable. 


\section{EL RECURSO SOLAR}

La estimación del recurso solar disponible en la vivienda se realizará en base los datos de la base de datos europea del PVGIS [1]. La vivienda tiene prevista una superficie inicial de unos $33 \mathrm{~m} 2$ colocados sobre el tejado de la vivienda (ver figura 8) orientados al sur (azimuth de $0^{\circ}$ ) y con ángulo de orientación definible por el estudiante. Por dar una idea orientativa del recurso solar disponible en la ubicación seleccionada, la figura 21, muestra un primer cálculo básico con una potencia instalada de $3.3 \mathrm{~kW}$ y un rendimiento de las conversiones electrónicas necesarias del 90\%. La inclinación óptima de los paneles sobre la horizontal es de $37^{\circ}$ y el sistema proporcionaría una energía anual de unos 4.14 MWh, con un mínimo mensual en el mes de diciembre, mes en el cual se producen unos $7.29 \mathrm{kWh} /$ día (unos $220 \mathrm{kWh} / \mathrm{mes}$ ).

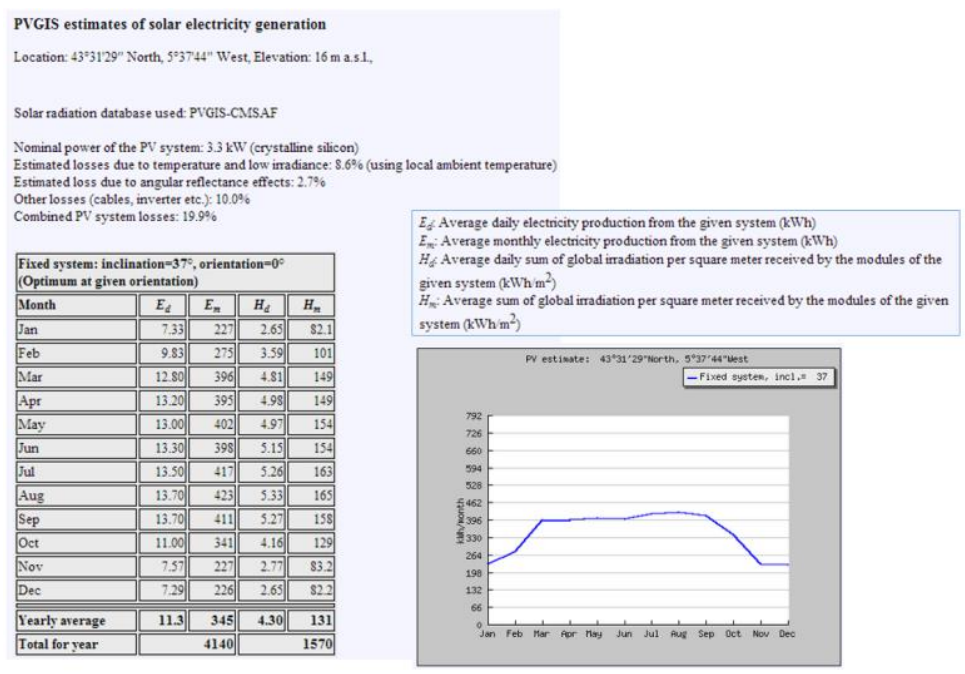

Fig. 21. Una primera estimación del recurso solar.

Instalación de zonas con energía solar adicionales a las previstas son también posible en las propuestas realizadas por los estudiantes, por ejemplo elementos de mobiliario urbano, marquesinas, puntos de carga de vehículos eléctricos, etc. En estos casos, diseños, orientaciones, superficies disponibles, etc quedan a la discrecionalidad del diseñador.

\section{EL RECURSO EÓLICO}

De una forma análoga la estimación del recurso eólico disponible para la vivienda se realizará a partir de Atlas eólico proporcionado por el IDEA [2]. Así la figura 23, recoge la velocidad media del viento en cada 
una de las estaciones del año y los parámetros de la distribución estadística Weibull de los mismos. Los datos están calculados a una altitud de $80 \mathrm{~m}$

Utilizando estos datos se ha estimado que el recurso eólico que aportaría una turbina de $30 \mathrm{~m}^{2}$ con un rendimiento del 35\% sería de unos $10 \mathrm{MWh}$. En la misma figura 24 se resumen los datos del cálculo realizado. Las correcciones de altitud se han realizado utilizando la Ley Exponencial de Hellmann con un coeficiente $\alpha$ de 0.13 que se corresponde con un terreno poco accidentado.

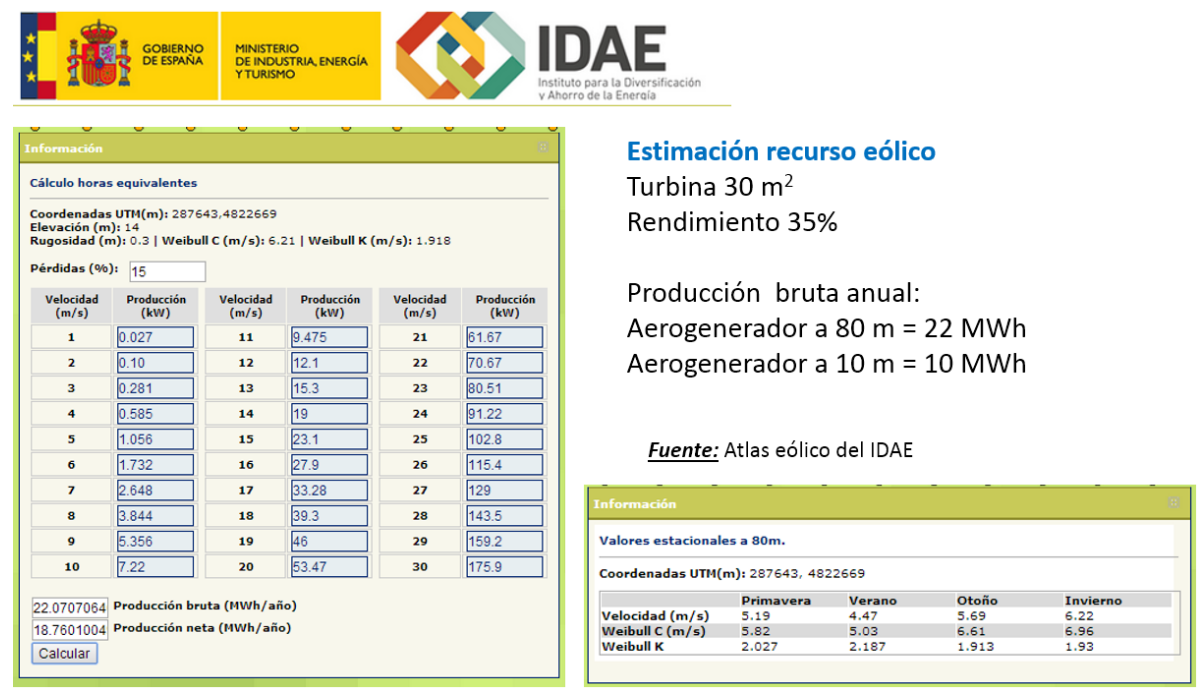

Fig. 23. Recurso eólico estimado con las herramientas del IDEA y extrapolados a $10 \mathrm{~m}$.

\section{OTRAS PROPUESTAS DE LINEAS DE TRABAJO}

Definida la vivienda patrón educacional (EPH), su ubicación geográfica, dimensiones geométricas, datos relativos a calefacción, ACS y definición de los recursos solar y eólico y líneas de trabajo derivadas de los mismos, se sugieren al estudiante una relación adicional de temas de trabajo, temas que pueden ser ampliados a iniciativa y creatividad del propio estudiante.

La figura 24 muestra un pequeño resumen de los cálculos realizados en este documento, resumiendo las necesidades energéticas para calefacción, agua caliente sanitaria (ACS) y pérdidas en el depósito de ACS, junto a los recursos renovables disponibles tanto eólica como solar. Se observa que con las propuestas realizadas el balance es positivo, es decir, es posible cubrir la demanda energética de la vivienda EPH utilizando fuentes renovables. La introducción de estrategias de ahorro de energía, nuevas fuentes renovables (p.e. Gimnasio o 
mobiliario urbano, etc) o cualquier otro tipo de mejora puede ser introducida en esta propuesta educacional.

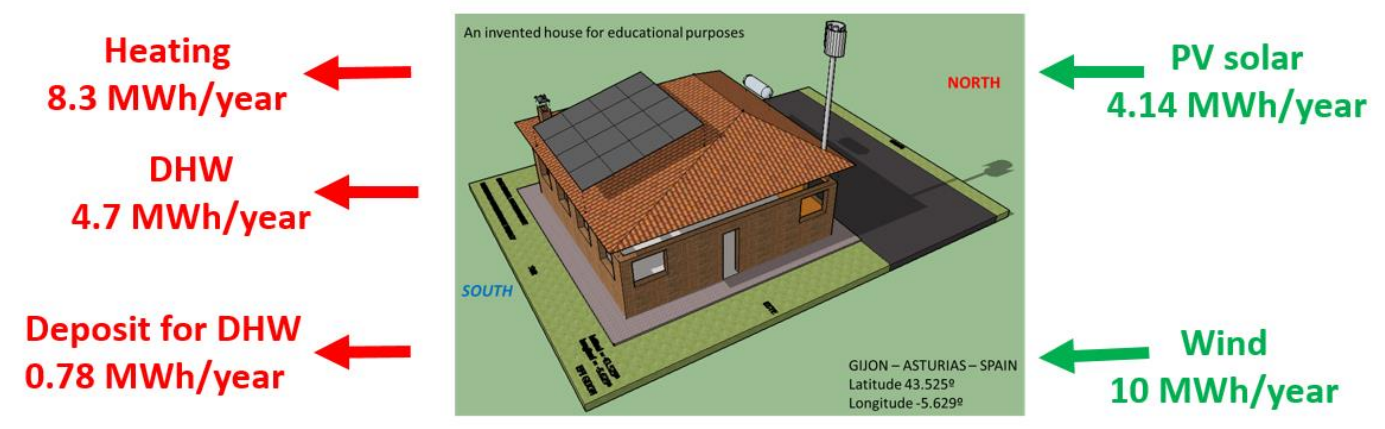

Fig. 24. Un pequeño resumen de las necesidades energéticas de la vivienda EPH y los recursos renovables disponibles

La vivienda patrón educacional (EPH) está propuesta con el único fin de desarrollar la creatividad y el aprendizaje del estudiante de ingeniería en temas de energías renovables, eficiencia energética y electrónica de potencia y entre los temas propuestos a los estudiantes podemos citar los siguientes:

(a).- Diseños de iluminación exterior e interior basados en diodos LED de alta eficiencia. Estrategias de ahorro de energía. [6]

(b).- Distribuciones en continua (baja tensión $48 \mathrm{~V}$ ) y alta tensión (400 V) para satisfacer las necesidades energéticas de la vivienda e incorporar nuevos servicios e ideas (Redes Inteligentes o Smart Grids). Se sugiere el estudio de convertidores continua-continua didireccionales con aislamiento galvánico como tema interesante en este campo (por ejemplo, el puente activo bidireccional "DAB converter" [5]).

(c).- Elementos inteligentes para mobiliario urbano (tanto en exterior como interior). Puntos de carga para móviles y ordenadores. Zonas con calefacción o aire acondicionado en el exterior.

(d).- Puntos de carga de vehículos eléctricos (EVs) aprovechando el recurso renovable disponible. [7]

(e).- Suavizado del pico de potencia demandado de la red. Gestión horaria inteligente de la energía consumida.

Gestión inteligente de la energía consumida en la vivienda y sus electrodomésticos o servicios que demanden consumo importante de energía.

(f).- Aprovechamiento del agua. Reutilización del agua de duchas y lavabos. Aprovechamiento del agua de lluvia.

(e).- Certificación energética de la vivienda patrón (EPH) a partir de la normativa nacional existente, utilizando el programa CE3X. [3] 
(g).- Nuevas fuente de generación de energías renovables. Energía generada en un gimnasio o zonas de juego que generen energía.

\section{CONCLUSIONES}

Se ha propuesto una vivienda patrón educacional $(\mathrm{EPH})$ como marco de trabajo predefinido para el aprendizaje de campos de actualidad como son las energías renovables, la electrónica de potencia y la eficiencia energética. Sobre esta hipotética vivienda predefinida en este documento se pueden desarrollar una gran variedad de proyectos de ingeniería una buena parte de ellos propuestos en este mismo documento.

\section{AGRADECIMIENTOS}

Todos estos trabajos están apoyados por el "Ministerio de Educación y Ciencia" del Gobierno de España mediante los proyectos (ENERLIGHT project- referencia MICINN-10-DPI2010-15889) y (LITCITY project referencia ENE 2013-41491-R). Se quiere agradecer el apoyo de todos los estudiantes y profesores que participantes en el Taller de Energías Renovables (Workroom on Renewable Energy -WRE) de la EPI de Gijón por toda la aportación de ideas y sugerencias recibidas para la elaboración de este documento.

\section{REFERENCIAS}

[1] Estimación del recurso solar. Base europea de datos de PVGIS (Photovoltaic Geographical Information System). http://re.jrc.ec.europa.eu/pvgis/apps4/pvest.php

[2] Estimación del recurso eólico. Altas eólico del IDEA. http://atlaseolico.idae.es/meteosim/

[3] Certificación energética de edificios. Programa CE3X.

http://www.idae.es/index.php/relcategoria.1030/id.718/relmenu.346/mod.pags/mem.detalle

[4] Garcia-Rodriguez, Segundo "Using Renewable Energy to provide Domestic Hot Water (DHW), Heating and Electric Power in an Isolated House”. Workrooms Journal. Issue 2. July 2014. ISSN: 2386-2483.

DOI: http://dx.doi.org/10.15592/workrooms.2014.0004

[5] Rico-Secades, Manuel; Garcia-Llera, Daniel; Lopez-Corominas, Emilio; Calleja-Rodriguez, Antonio Javier. "Designing Dual-Active Bridge (DAB) Converter for Energy Storage/Recovery Systems in a Lighting Smart 
Grid context". Workrooms Journal. Issue 2. July 2014. ISSN: 2386-2483. DOI: http://dx.doi.org/10.15592/workrooms.2014.0003

[6] M. Jaureguizar, D. Garcia-Llera, M. Rico-Secades, A.J. Calleja, E.L. Corominas. "Enerlight project: Walking from electronic lighting systems to Lighting Smart Grid”. IEEE SmartMILE 2013 Conference. December 2013. DOI :10.1109/SmartMILE.2013.6708166

[7] Escandón-López, Javier; Moro-Pérez, Gonzalo. “Charge System for EVs”. Workrooms Journal. Issue 2. July 2013. ISSN: 2386-2483. DOI: http://dx.doi.org/10.15592/workrooms.2013.0004 